\title{
Foreword
}

\section{Mapping the Heart}

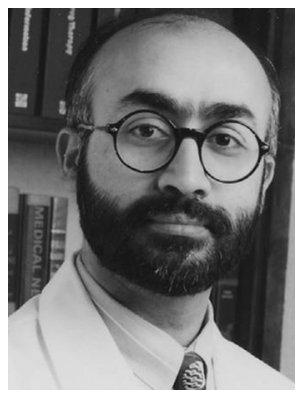

Ranjan K. Thakur, MD, MPH, MBA, FHRS

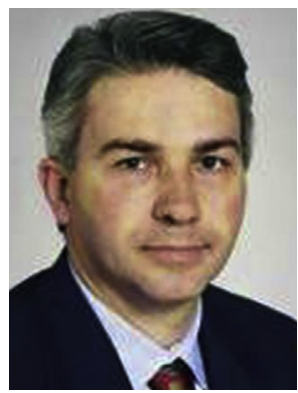

Andrea Natale, MD, FACC, FHRS

Consulting Editors

Cardiac mapping is simply the correlation of the electrical activity of the heart to the underlying anatomy. In its simplest form, placement of multipolar intracardiac catheters and induction of sustained arrhythmias are a form of mapping to determine the location of an accessory pathway or the site of origin of ventricular tachycardia, and such. Since interventional electrophysiology began in the 1980s, more and more sophisticated cardiac mapping tools as well as electrophysiologic concepts have been developed to pinpoint the mechanisms and the exact anatomic localization of susceptible arrhythmic substrates. Cardiac mapping has not been fully automated, but requires detailed electrophysiology study, the use of electrophysiologic maneuvers, and deductive reasoning.

Dr Shenasa has been active for over 2 decades in summarizing the advancements in cardiac mapping periodically. His current book, Cardiac Mapping is in its fifth edition. We are grateful to Drs Shenasa and Al-Ahmad for their effort of providing a summary of contemporary issues of interest in cardiac mapping for the clinical electrophysiology community. They have assembled an international panel of experts to discuss everything from anatomical considerations in mapping, the fundamentals of cardiac mapping, and unipolar mapping, all the way to the comparative advantages of the commercially available mapping systems. This issue contains useful information for electrophysiology fellows, associated professionals in electrophysiology as well as the practicing electrophysiologist.

We hope the readership will find this issue useful and informative.

Ranjan K. Thakur, MD, MPH, MBA, FHRS Sparrow Thoracic and Cardiovascular Institute Michigan State University 1200 East Michigan Avenue, Suite 580 Lansing, MI 48912, USA

Andrea Natale, MD, FACC, FHRS Texas Cardiac Arrhythmia Institute

Center for Atrial Fibrillation at

St. David's Medical Center 1015 East 32nd Street, Suite 516 Austin, TX 78705, USA

E-mail addresses:

thakur@msu.edu (R.K. Thakur) andrea.natale@stdavids.com (A. Natale) 